\title{
Gerenciamento de demanda em redes elétricas inteligentes usando sistemas multiagentes
}

\author{
J. V. M. Roma*. D. Q. Oliveira*. F. M. Portelinha Jr**, Shigeaki Leite de Lima* \\ *Universidade Federal do Maranhão \\ SãoLuís,Brasil(jvmr20@gmail.com,dq.oliveira@ufma.br, shigeaki.lima@ufma.br). \\ **Instituto Nacional de Telecomunicações \\ Santa Rita do Sapucaí, Brasil (portelinha@gmail.com)
}

\begin{abstract}
The smart grids allow more interactivity between consumers and power utility, increasing the consciousness regarding energy efficiency. The application of distributed control is promising in this new grid, such as multiagent systems. This paper presents the application of multiagent systems to solve demand management in power systems. The agent has previous information on the power demand and the prices, and by applying an evolutionary algorithm, it can propose the best way to use energy to minimize the costs. Two different cases of dynamic pricing are investigated, and the results are described, showing the possibility to apply the proposed methodology.
\end{abstract}

Resumo: As redes elétricas inteligentes proporcionam maior interatividade entre os consumidores e a concessionária, aumentando a consciência do primeiro em relação ao uso eficiente da eletricidade. Neste contexto, a aplicação de sistemas de controle distribuídos parece ser promissora, destacando-se a aplicação de sistemas multiagentes para diversas tarefas. Este artigo apresenta a aplicação de sistemas multiagentes para solução do problema de gerenciamento de demanda em redes elétricas. O agente possui informações prévias sobre o uso de eletricidade do consumidor e dos preços das tarifas, e usando um algoritmo evolutivo, ele é capaz de propor o melhor uso da eletricidade de forma a minimizar os custos mensais. Dois casos diferentes de tarifação horária são investigados e os resultados são descritos, mostrando a possibilidade de aplicação da metodologia proposta.

Keywords: - multiagent systems, demand management, genetic algorithms.

Palavras-chaves: Sistemas multiagentes, gerenciamento de demanda, algoritmos genéticos.

\section{INTRODUÇÃO}

As redes elétricas inteligentes proporcionam possibilidades de maior interatividade entre consumidores e concessionárias de energia elétrica, conscientizando a sociedade sobre a necessidade de usar a eletricidade de forma eficiente. O Gerenciamento de Demanda (DSM) é considerado a solução para o problema clássico de dimensionamento do sistema elétrico, ou seja, o fato de que a infraestrutura é projetada para acomodar a demanda de pico, fazendo com que o sistema opere grande parte do tempo com capacidade ociosa. E atualmente com o crescimento da carga e a limitação na capacidade de investimentos, a demanda máxima projetada pode ser excedida e a concessionária ter problemas de qualidade de energia (Dehghanpour et al, 2018).

A resposta à demanda é definida como "mudanças no comportamento de clientes de uso final de seus padrões de consumo normais em resposta à mudanças no preço da eletricidade ao longo do tempo ou a incentivos destinados a induzir o menor consumo de eletricidade em momentos de altos preços no mercado atacadista, ou em momentos em que a confiabilidade do sistema é comprometida" (Hamilton and Gulhar, 2010). Ao se aplicar o DSM em um sistema elétrico, percebe-se uma mudança na operação do mesmo por meio do uso mais racional dos recursos disponíveis, atingindo assim menores perdas elétricas, menos congestionamento das linhas em horários de pico e maior fator de carga.

A maneira tradicional de implementar o DSM é através de tarifação dinâmica, ou seja, baixando as tarifas em momentos em que a demanda agregada deve ficar abaixo da média de modo a incentivar o usuário final a transferir suas cargas flexíveis para esses períodos. Outra maneira é através de programas baseados em incentivos, onde os consumidores que aderem ao programa de gerenciamento recebem incentivos monetários em troca de serviços auxiliares (Saffre and Gedge, 2010; Dehghanpour et al, 2018).

O gerenciamento de demanda é investigado em (Samadi et al., 2012) onde os autores propõem uma abordagem que busca gerenciar a demanda de acordo com objetivos sociais, ou seja, que o bem-estar do consumidor não seja afetado. Para tanto desenvolvem uma ferramenta de mechanism design (VickreyClarke-Groves mechanism) capaz de maximizar uma função utilidade para cada consumidor, reduzindo assim sua demanda sem afetar seu bem-estar.

Quian et. al (2013) apresentam um esquema de preços em tempo-real que reduz a razão entre o pico de demanda e a 
demanda media através de uma interação continua entre os consumidores e a concessionária de energia. De um lado o usuário maximiza seu excedente (diferença entre a quantidade usada e o preço pago) enquanto do outro lado a concessionária usa um esquema de precificação que tenta maximizar seus lucros. Através dessa interação contínua em tempo real ambos os envolvidos atingem seus objetivos. Safdarian et al. (2014) apresentam uma proposta de precificação em tempo real capaz de aumentar o lucro da concessionária de energia enquanto também apresenta bons resultados no gerenciamento de demanda usando um modelo de otimização inteira-mista.

O gerenciamento de demanda tem uma natureza distribuída, pois existem milhares de consumidores com diferentes características conectados ao sistema. Assim, a aplicação de sistemas multiagentes ao problema de gerenciamento de demanda é mais apropriada e tem sido bastante investigada na literatura. Por meio de diversas técnicas computacionais e comunicação ponto-a-ponto, esses agentes decidem e aprimoram a operação dos subsistemas trazendo benefícios a todos.

Dehghanpour et. al. (2018) estudam o comportamento do preço no mercado de curto prazo em um Sistema de distribuição inteligente. Enquanto os agentes dos consumidores empregam um algoritmo $Q$-learning para otimizar o seu padrão de consumo usando como parâmetros o preço da eletricidade e o conforto do usuário, o agente da concessionária usa técnicas de aprendizado de máquina para prever o comportamento dos consumidores e assim reduzir as incertezas no processo de determinação das tarifas de eletricidade.

Colson e Nehrir (2013) e Netto et. al. (2018) também aplicam sistemas multiagentes no gerenciamento de microrredes. Os sistemas de informação e a infraestrutura de comunicação necessários para fazer esse sistema distribuído funcionar corretamente são investigados em (Netto et al., 2018). Santos et al. (2018) investigam a aplicação de sistemas multiagentes para gerenciamento de operação de microrredes em modo conectado à rede. Outras aplicações de sistemas multiagentes para microrredes resilientes são descritas em Dehghanpour et al. (2017).

Este artigo propõe investigar o gerenciamento de demanda em duas situações distintas. Na primeira situação existe um esquema de tarifação horária com três postos tarifários diferentes durante o dia. Na segunda situação, a tarifa é dinâmica e depende da demanda do alimentador. Um diferencial deste trabalho é a utilização de uma descrição microeconômica do problema, tornando a metodologia mais aderente às situações reais. O objetivo é descrever e verificar o comportamento dos agentes em ambas as situações, demonstrando o funcionamento da metodologia proposta e os resultados obtidos.

\section{GERENCIAMENTO DE DEMANDA EM REDES ELÉTRICAS INTELIGENTES}

De acordo com Dehghanpour et al. (2018), a ideia básica por trás do gerenciamento de demanda é introduzir mecanismos de mercado a nível de distribuição, onde agentes automatizados são capazes de responder a variações de tarifas de eletricidade. Dessa forma, ao modificar o preço do produto é possível influenciar na procura do mesmo, levando a curva Procura x Oferta a um novo ponto de equilíbrio.

Sob o ponto de vista microeconômico, podemos usar a equação de Slutsky (1) para analisar o bem energia elétrica (Varian, 2005):

$$
\Delta x_{1}=\Delta x_{1}^{s}+\Delta x_{1}^{n}
$$

De acordo com (1), a quantidade demandada de um bem é a soma de dois efeitos: o de substituição $\left(\Delta x_{1}^{S}\right)$ e da receita $\left(\Delta x_{1}^{n}\right)$. O efeito de substituição indica como o consumidor substitui um bem por outro quando há uma variação no preço, mas o poder de compra permanece o mesmo. Mais precisamente, $\Delta x_{1}^{S}$ é a taxa de mudança na demanda do produto quando o preço do mesmo varia para $p_{1}^{\prime}$, e ao mesmo tempo, o poder de compra permanece igual (2) (Varian, 2005).

$$
\Delta x_{1}^{S}=x_{1}\left(p_{1}^{\prime}, m^{\prime}\right)-x_{1}\left(p_{1}, m\right)
$$

Já o efeito da receita mostra que a demanda por um bem aumenta quando seu preço cai, desde que este bem seja normal, sendo representada em (3):

$$
\Delta x_{1}^{n}=x_{1}\left(p_{1}^{\prime}, m\right)-x_{1}\left(p_{1}^{\prime}, m^{\prime}\right)
$$

Assim, substituindo (2) e (3) em (1), temos (4):

$$
\Delta x_{1}=x_{1}\left(p_{1}^{\prime}, m\right)-x_{1}\left(p_{1}, m\right)
$$

a qual mostra que a demanda por um produto aumenta quando a receita aumenta, e que a demanda por esse produto diminui quando o preço do mesmo aumenta. Sabe-se, no entanto, que o preço que o consumidor deseja pagar para consumir determinado bem depende da quantidade de recursos financeiros disponíveis para consumir outros bens. Assim, ao aumentar os preços da energia elétrica para gerenciar a demanda, a redução no consumo se deve mais às restrições orçamentárias do que propriamente a aceitação do corte de energia (Harris, 2006).

\subsection{Abordagem usando sistemas multiagentes}

A Figura 1 apresenta de forma mais didática o problema do gerenciamento de demanda sob a ótica de sistemas multiagentes. A concessionária está representada no quadro por um agente, assim como diferentes consumidores são representados por seus respectivos agentes. Conforme visto na Figura, podem existir consumidores não dispostos a aderir ao programa de gerenciamento de demanda da concessionária, se dispondo a pagar tarifas mais elevadas para consumo da eletricidade em qualquer momento.

O agente é uma entidade computacional que possui características como inteligência, proatividade, sociabilidade e hierarquias. Assim, cada agente busca permanentemente seus próprios objetivos por meio de decisões inteligentes e troca de mensagens com os agentes próximos. Em situações de emergência, os agentes podem cooperar entre si de forma a manter o sistema funcionando (McArthur et al., 2007, GomezSanz et al., 2014). 


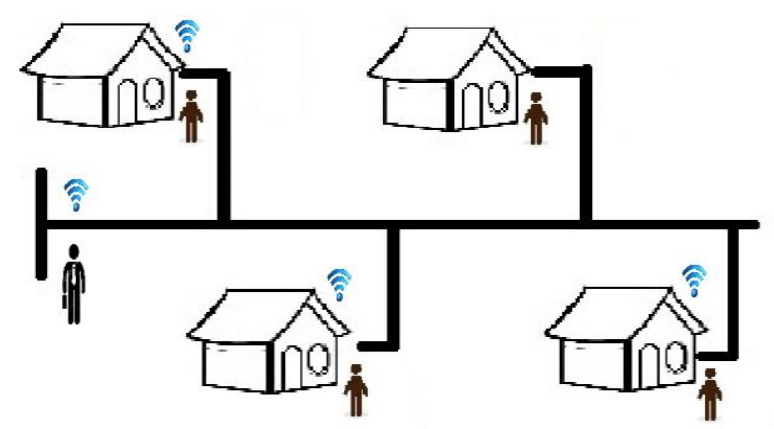

Fig. 1.Alimentador de distribuição com seus respectivos agentes

Por meio de mensagens enviadas aos consumidores, a concessionária informa as tarifas vigentes de eletricidade. Assim, cada agente é capaz de planejar o melhor uso da energia elétrica de forma a minimizar os custos, além de atender outros objetivos de seu proprietário. Em determinados momentos, a concessionária pode enviar pedidos de corte de carga aos consumidores inscritos no programa de gerenciamento de demanda, estabelecendo um controle de caráter distribuído em toda a rede elétrica.

\subsection{Comunicação entre os agentes}

Para atingir seus objetivos, os agentes se comunicam entre si, enviando pedidos, perguntas e informações aos seus pares e atuando sobre os dispositivos controlados pelos mesmos após a tomada de decisão. Logo, os sistemas de comunicação são imprescindíveis para o pleno funcionamento dos sistemas multiagentes, e, por conseguinte, para o gerenciamento das redes elétricas inteligentes.

Também é necessário a definição do tipo de linguagem de comunicação entre os agentes (ACL - Agent Communication Language), cujo objetivo é a de troca de informação e a consolidação da sintaxe entre eles. Por meio da definição do tipo de comunicação entre estes agentes, o agente consumidor irá conversar com o agente concessionária para realizar a operações necessárias para a contingência do Sistema.

As tecnologias de comunicação existentes podem ser com fio (wired) ou sem fio (wireless). As comunicações com fio, baseadas em telefonia fixa e fibra óptica, são normalmente utilizadas em aplicações de largura de banda estreita, sendo inviáveis quando existirem grande número de dispositivos conectados em uma grande área geográfica. Para este segundo caso, as redes sem fio são mais indicadas (Gungor et. al., 2013).

Dentre as tecnologias cabeadas pode-se citar o Power Line Communication (PLC) e a fibra óptica. Já dentre as tecnologias wireless pode-se citar o Bluetooth, Wi-Fi, ZigBee, Long Term Evolution (LTE - 4G), LoRa, WiMax, comunicação via satélite, dentre outras. A escolha da tecnologia de comunicação depende das necessidades de cada aplicação, tais como distância, qualidade de serviço (QoS), latência, confiabilidade e taxa de dados. Maiores informações sobre cada uma dessas tecnologias são descritas em (Zambroni de Souza et al., 2017; Portelinha Jr. et al. 2017).

Dentre as tecnologias de baixo alcance úteis para aplicações em Home Area Network (HAN), pode-se citar o Wi$F i$ e o Bluetooth. Estas tecnologias podem ser usadas para comunicação entre os medidores inteligentes e outros dispositivos no âmbito doméstico, pois possuem baixo alcance e baixo custo de implementação. Quando se parte para a comunicação externa (Neighborhood Area Network - NAN) entre os diferentes agentes, a comunicação pode ser feita por LTE, WiMax ou LoRa. As vantagens estão na cobertura de grandes áreas e de milhares de usuários, embora possuam um custo de infraestrutura considerável (Gungor et al., 2013). Tais tecnologias wireless são propícias para esta finalidade, não restringindo a possibilidade de uso de mais de uma delas ou de redes híbridas usando tecnologias com cabo e sem cabo.

\section{METODOLOGIA PROPOSTA}

A proposta desse artigo se insere em um contexto de redes elétricas inteligentes, onde diferentes agentes trocam informações em tempo real por meio de uma rede de informações dedicada apropriada para tal aplicação. Considera-se que cada consumidor é representado por um agente, que passa a ser chamado de Agente Consumidor. Este agente gerencia um sistema doméstico de cargas, e conhecendo o perfil de uso diário de eletricidade, pode propor mudanças de forma a minimizar o custo da energia elétrica.

Outro elemento importante da proposta é o Agente Concessionária, que representa a concessionária de energia elétrica e que monitora o fluxo de energia nos alimentadores durante o dia. Ele é responsável por enviar sinais aos consumidores que aderiram ao programa de gerenciamento da demanda da concessionária para que diminuam o consumo de energia elétrica em determinadas situações ou horas do dia em troca da redução no valor da conta, devido ao consumo em horários de tarifa mais baixas quando a rede está menos congestionada.

Duas abordagens são investigadas neste trabalho. A primeira abordagem é o gerenciamento de demanda por meio da tarifação horária, onde são adotados três postos tarifários durante os dias úteis: uma tarifa menor para o horário fora de ponta, uma tarifa intermediária aplicada a partir de duas horas antes da ponta e até uma hora depois da ponta; a tarifa de ponta, aplicada durante três horas seguidas do dia. Este esquema se assemelha ao que a Agência Nacional de Energia Elétrica propôs implementar por meio da tarifa branca aos consumidores residenciais (ANEEL, 2019).

Em ambos os cenários, o Agente Concessionária informa no início do dia os horários das tarifas, e o Agente Consumidor é capaz de planejar a utilização da energia elétrica por meio de um algoritmo genético, determinando o estado (ligado/desligado) de cada um dos utensílios ou circuitos dentro da residência a cada faixa horária do dia. Para executar tal tarefa, ele deve saber previamente as tarifas horárias que são informadas pelo Agente Concessionária.

As cargas residenciais são classificadas em fixas e móveis, de acordo com a preferência do usuário para cada horário. A partir daí se estabelecem restrições de tempo mínimo de funcionamento que devem ser obedecidas pelo Agente Consumidor para planejamento do uso da energia elétrica. A configuração do agente pode ser feita previamente de forma manual pelo proprietário, ou ele pode aprender sozinho essas preferências por meio de técnicas inteligentes já descritas na literatura (López et al., 2019; Basu et al., 2013; Goel et al., 2007). Aqui se preferiu considerar que o usuário configura o 
Agente. A decisão de seguir o plano ótimo é dada ao proprietário, cabendo ao Agente Consumidor nesse caso apenas sugerir um plano otimizado de uso da eletricidade.

A otimização realizada pelo Agente Consumidor é definida em (5), onde o objetivo é minimizar os custos de uso de eletricidade. Por meio desse processo de otimização é que todos os $\mathrm{U}_{\mathrm{i}, \mathrm{j}}$ são determinados em cada intervalo de tempo. Assim, U é uma matriz de números binários com dimensão $m$ $\mathrm{x}$. .

$$
\operatorname{Min} C=\sum_{i=1}^{n} \sum_{j=1}^{m} T_{i} \cdot U_{i, j} \cdot P_{j}
$$

onde: i é o intervalo do dia; $n$ é o número total de intervalos em que o dia foi dividido; $\mathrm{T}_{\mathrm{i}}$ é a tarifa de eletricidade aplicada no horário $\mathrm{i}$; $\mathrm{j}$ é o utensílio elétrico dentro de um conjunto de m utensílios existentes; e $U_{i, j}$ é o estado do utensílio j no horário i do dia, podendo estar ligado ou desligado a cada horário; $\mathrm{P}_{\mathrm{j}}$ é a potência consumida pelo utensílio elétrico $\mathrm{j}$.

A segunda abordagem é a da tarifação dinâmica baseada na demanda do sistema. Neste caso, o reajuste realizado pelo Agente Concessionária segue as seguintes regras: (1) Existem três postos tarifários; (2) O Agente Concessionária monitora a demanda total do sistema, mantendo um registro das demandas máxima e mínima já alcançadas e, sempre que a demanda total do sistema ficar acima de $2 / 3$ do intervalo entre a demanda máxima e mínima, a tarifa é reajustada para o valor do posto tarifário mais caro, mesmo que isto ocorra fora do horário na qual esta tarifa é prevista. Assim que a demanda diminuir e ficar entre $1 / 3$ e $2 / 3$ do intervalo, a tarifa é reajustada para a intermediária. Se a demanda total ficar abaixo de $1 / 3$ do intervalo, a tarifa é reajustada para o posto tarifário mais barato; (3) O Agente Concessionária pode enviar pedidos de corte de carga no posto tarifário mais caro aos consumidores que aderiram ao contrato de gerenciamento de demanda, a fim de diminuir a demanda naquele horário, e aviso de que podem voltar a consumir durante o posto tarifário mais barato.

Nesta segunda abordagem o Agente Consumidor se comporta para responder às mudanças de tarifa e aos pedidos de corte de carga enviados pelo Agente Concessionária. Assim, o Agente Consumidor observa a prioridade de cada carga individual, desligando a mesma se esta estiver ligada para ser usada em outro horário de tarifa mais barata caso o Agente Concessionária tenha enviado a ordem de reduzir a demanda.

O Agente Consumidor agora também incorpora regras heurísticas além daquelas descritas para a primeira abordagem, de modo a se adaptar às mudanças de tarifa. As decisões são tomadas de acordo com regras estabelecidas conforme descritos abaixo:

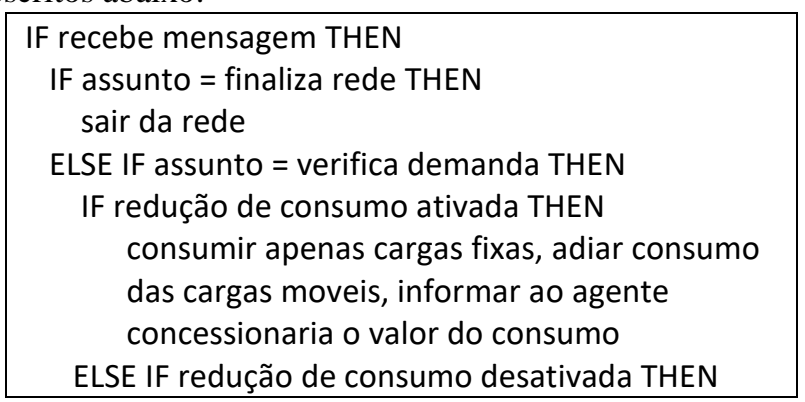

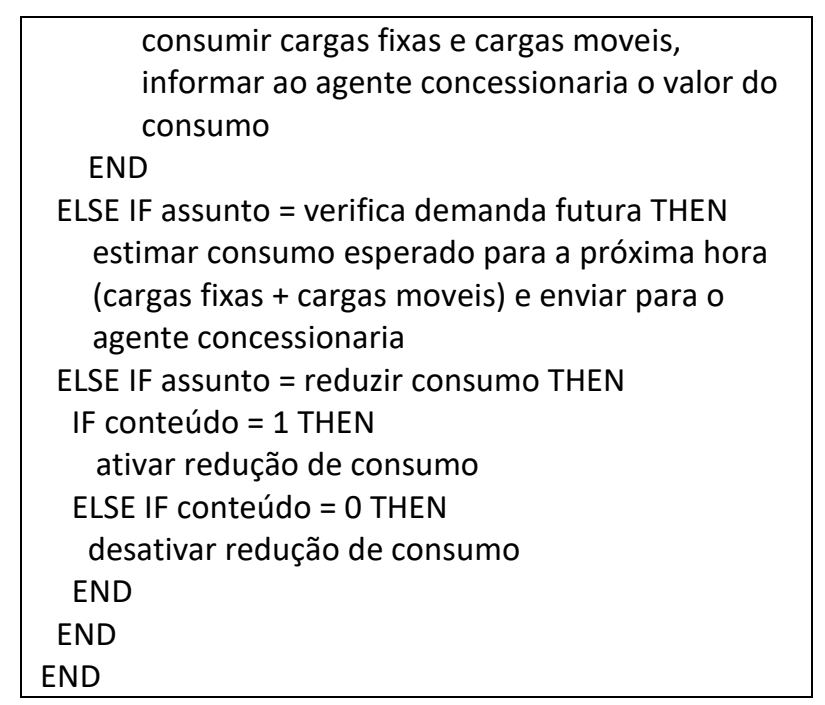

\section{ESTUDO DE CASO}

A metodologia proposta para gerenciamento de demanda em redes inteligentes foi testada em um sistema com as características descritas a seguir. Foram considerados dez consumidores pertencentes a cinco categorias diferentes, ou seja, de perfis de consumo variado. Os consumidores A, B, C e D são residenciais, enquanto o consumidor E é uma pequena empresa. Cada um desses clientes é representado no problema por um Agente Consumidor que conhece e propõe um perfil otimizado de uso de eletricidade para o mesmo. Alguns desses clientes participam do programa de gerenciamento de demanda, enquanto outros não aderiram ao programa proposto. Informações sobre as tarifas aplicadas ao problema e as cargas de cada consumidor são dadas abaixo, respectivamente, nas Tabelas 1 e 2 .

TABELA 1 - TARIFAS DE BAIXA TENSÃO

\begin{tabular}{|c|c|c|c|}
\hline $\begin{array}{c}\text { Classes de } \\
\text { consumidores }\end{array}$ & $\begin{array}{c}\text { Horário de } \\
\text { ponta }\end{array}$ & $\begin{array}{c}\text { Horário } \\
\text { intermediário }\end{array}$ & $\begin{array}{c}\text { Horário } \\
\text { Fora de } \\
\text { ponta }\end{array}$ \\
\hline $\begin{array}{c}\text { Residencial/ } \\
\text { Comercial }\end{array}$ & $\begin{array}{c}1,40 \\
\mathrm{R}\end{array} / \mathrm{KWh}$ & $0,88 \mathrm{R} \$ / \mathrm{kWh}$ & $0,52 \mathrm{R} \$ / \mathrm{kWh}$ \\
\hline
\end{tabular}

TABELA 2 - CARACTERÍSTICAS DOS CONSUMIDORES

\begin{tabular}{|c|c|c|c|c|}
\hline $\begin{array}{c}\text { Classe do } \\
\text { consumidor }\end{array}$ & $\begin{array}{c}\text { Consumo } \\
\text { mensal } \\
(\mathrm{kWh})\end{array}$ & $\begin{array}{c}\text { Proporção } \\
\text { de cargas } \\
\text { fixas (\%) }\end{array}$ & $\begin{array}{c}\text { Proporção } \\
\text { de cargas } \\
\text { móveis } \\
(\%)\end{array}$ & Quantidade \\
\hline A & 99 & 77,88 & 22,12 & 2 \\
\hline B & 192 & 66,67 & 33,33 & 2 \\
\hline C & 685 & 78,18 & 21,82 & 2 \\
\hline D & 1099 & 78,05 & 21,95 & 2 \\
\hline E & 1418 & 66,68 & 33,32 & 2 \\
\hline
\end{tabular}

O Agente Consumidor aplica um Algoritmo Genético ao problema de otimização, determinando o perfil ótimo de uso das cargas que minimiza os custos de eletricidade. O Algoritmo genético utilizado possui uma população de 100 indivíduos, taxa de mutação de $4 \%$, taxa de crossover de $77 \%$ e 23\% de elitismo, seguindo recomendação de Linden (2012). O mesmo é executado até que não haja alterações no resultado durante 1000 gerações. 
Os resultados para a demanda do sistema para os casos com e sem gerenciamento podem ser observados nas Figuras 2 e 3. Os resultados obtidos para o teste mostram uma capacidade do Agente Consumidor determinar um perfil de uso otimizado minimizando a carga do consumidor. Além disso, é possível ver que a demanda do alimentador da concessionária também diminui quando se compara o caso sem gerenciamento da demanda. Um maior detalhamento nos resultados obtidos é mostrado na Tabela 3, onde se comparam os custos com a compra de eletricidade antes e depois da aplicação do algoritmo de gerenciamento de demanda para o caso de postos tarifários fixos. Observa-se uma redução nos custos com compra de eletricidade da ordem de 14,86\%, 21,60\%, 29,74\%, $29,74 \%$, e $12,97 \%$. A redução total da demanda no sistema chega a $21,60 \%$.

A Figura 4 ilustra a comunicação entre dois agentes do sistema, um Agente Consumidor e o Agente Concessionária, mostrando a troca de mensagens entre eles para que o segundo chegue a sua decisão final. Como neste caso estamos nos referindo ao caso de tarifação dinâmica, o Agente Consumidor apenas segue a determinação do Agente Concessionária.

Os resultados demonstram a eficácia do gerenciamento de demanda em sistemas elétricos inteligentes. Estudos de caso posteriores devem levar em conta modelos mais detalhados de satisfação do cliente, pois assim pode-se chegar a resultados mais realistas.

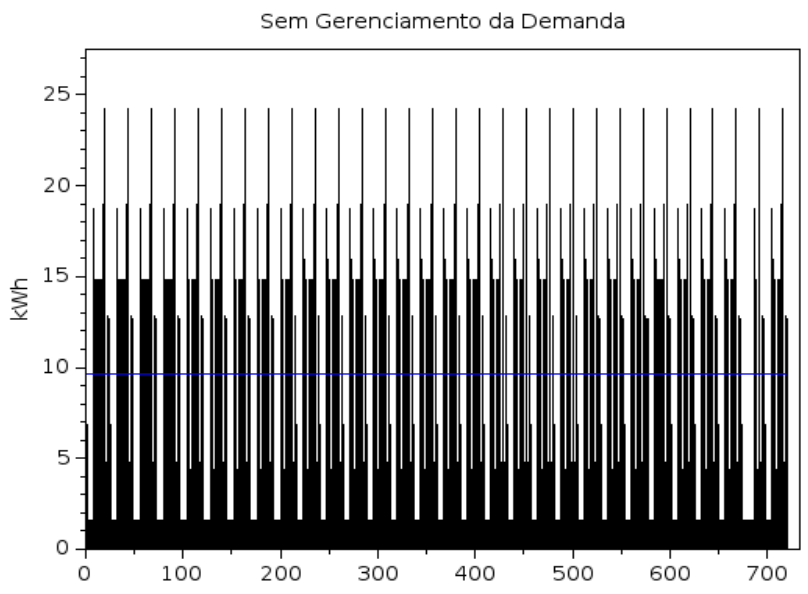

Fig. 2. Demanda do sistema sem gerenciamento

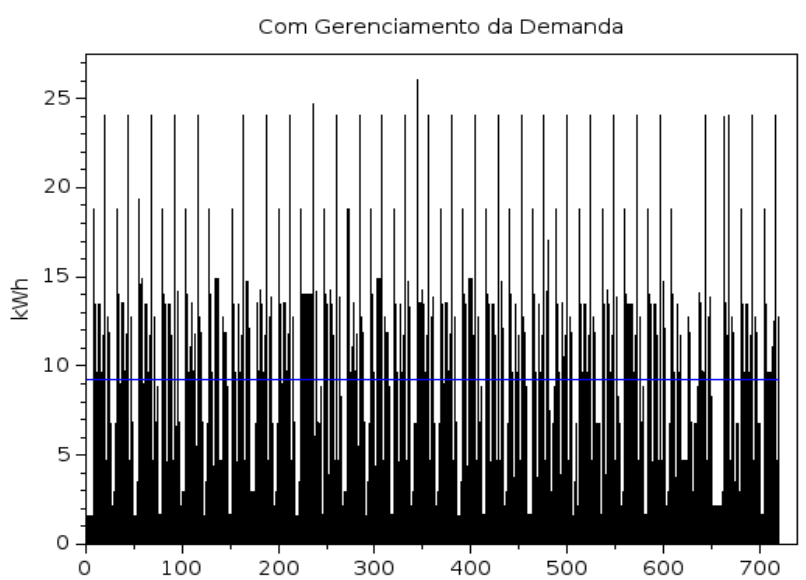

Fig. 3. Demanda do sistema com gerenciamento
TABELA 3 - COMPARAÇÃO ENTRE OS CASOS COM GERENCIAMENTO E SEM GERENCIAMENTO

\begin{tabular}{|c|c|c|}
\hline $\begin{array}{c}\text { Classe do } \\
\text { consumidor }\end{array}$ & $\begin{array}{c}\text { Custos sem } \\
\text { gerenciamento de } \\
\text { demanda (R\$) }\end{array}$ & $\begin{array}{c}\text { Custos com } \\
\text { gerenciamento de } \\
\text { demanda (R\$) }\end{array}$ \\
\hline $\begin{array}{c}\text { Consumidor } \\
\text { A }\end{array}$ & 70,95 & 60,41 \\
\hline $\begin{array}{c}\text { Consumidor } \\
\text { B }\end{array}$ & 144,23 & 113,08 \\
\hline $\begin{array}{c}\text { Consumidor } \\
\text { C }\end{array}$ & 538,45 & 378,31 \\
\hline $\begin{array}{c}\text { Consumidor } \\
\text { D }\end{array}$ & 845,12 & 593,75 \\
\hline Consumidor E & 872,92 & 759,73 \\
\hline
\end{tabular}

\section{CONCLUSÃO}

$\mathrm{O}$ artigo apresentou metodologias de gerenciamento de demanda baseada em sistemas multiagentes. Cada agente é responsável por propor um perfil de uso otimizado que minimize os custos de energia ao fim do mês e mantenha diretrizes de tempos de utilização mínimos dados pelo usuário. Os resultados comprovam a eficácia do método para se atingir a diminuição dos custos de energia e os efeitos positivos no carregamento do sistema elétrico. Estudos posteriores devem adicionar maiores detalhes no modelo de satisfação dos clientes, visto que esse aspecto pode modificar significantemente os resultados.

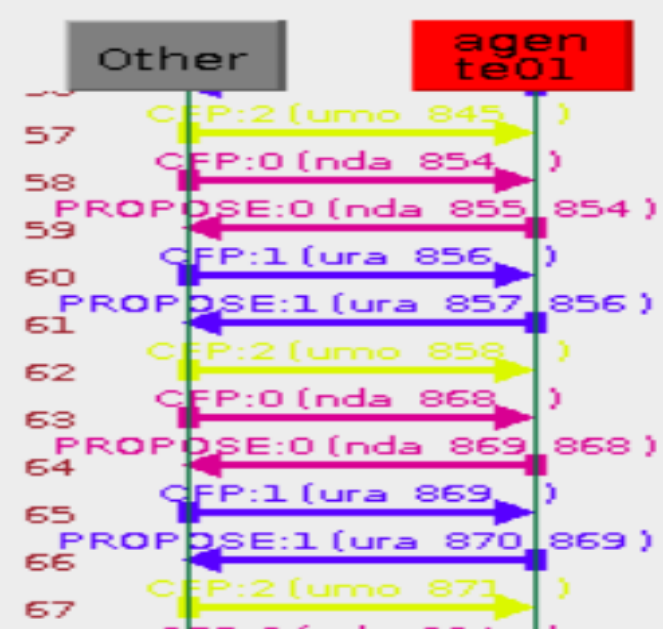

Fig. 4. Mensagens entre um Agente Consumidor e o Agente Concessionária

\section{AGRADECIMENTOS}

Os autores agradecem a FAPEMA por seu apoio financeiro para a execução deste trabalho.

\section{REFERENNCIAS}

Agência Nacional de Energia Elétrica - ANEEL. (2019). http://www.aneel.gov.br/tarifa-branca. Consultado em 08.02.2019.

Basu, K., Debusschere, V., Bacha, S. (2013). Residential appliance identification and future usage prediction from smart meter. 39th Conference of the IEEE Industrial Electronics Society, pp. 4994 - 4999. 
Colson, C. M., Nehrir, M. H. (2013). Comprehensive RealTime Microgrid Power Management and Control with Distributed Agents. IEEE Transactions on Smart Grid, v. 4, No. 1, pp. 617-627.

Dehghanpour K., Nehrir, M. H., Sheppard, J. W., Kelly, N.C. (2018) Agent-based modeling of retail electrical energy markets with demand response. IEEE Transactions on Smart Grid, V. 9, No. 4, pp. 3465 - 3475.

Dehghanpour, K., Colson, C., Nehrir, H. (2017). A Survey on Smart Agent-Based Microgrids for Resilient/Self-healing Grids. Energies, 10, 620.

Goel, L., Aparna, V. P., Wang, P. (2007). A Framework to Implement Supply and Demand Side Contingency Management in Reliability Assessment of Restructured Power Systems. IEEE Transactions on Power Systems, V. 22, No. 1, pp. 205-212.

Gomez-Sanz, J. J., Garcia-Rodriguez, S., Cuartero-Soler, N., Hernandez-Callejo, L. (2014). Reviewing Microgrids from a Multi-Agent Systems Perspective. Energies, 7, 3355-3382.

Gungor, V. C. et. al. (2013). A survey on smart grid potential applications and communications requirements. IEEE Transactions on Industrial Informatics, V. 9, No. 1, pp. $28-42$.

Hamilton, K., Gulhar, N. (2010) Taking Demand Response to the Next Level. IEEE Power \& Energy Magazine, pp. 6065.

Harris, Chris. (2006) Electricity Markets: Pricing, Structures and Economics. John Wiley \& Sons.

Linden, R. (2012). Algoritmos Genéticos. Ciência Moderna.

López, J. M. G., et al. (2019). Smart Residential Load Simulator for Energy Management in Smart Grids. IEEE Transactions on Industrial Electronics, V. 66, No. 2, pp. 1443-1452.

McArthur, S. D. J., Davidson, E. M., Catterson, V. M. et al. (2007). Multi-Agent Systems for Power Engineering Applications - Part 1: Concepts, Approaches, and Technical Challenges. IEEE Transactions on Power Systems, V. 22, No. 4, pp. 1743-1752.

Netto, R. S. et. al. (2018) Real-Time Framework for Energy Management System of a Smart Microgrid Using Multiagent, Systems. Energies, No. 11, 656.

Nyeng, P., Ostergaard, J. (2011). Information and Communications Systems for Control-by-Price of Distributed Energy Resources and Flexible Demand. IEEE Transactions on Smart Grid, V.2, No. 2, pp. 334341.

Portelinha Jr, F. M. et. al. (2017). Control Strategies for Improving Energy Efficiency and Reliability in Autonomous Microgrids with Communication Constraints. Energies, 10, pp. 1443.

Qian, L. P., Jun Zhang, Y., Huang, J., Wu, Y. (2013) Demand Response Management via Real-Time Electricity Price Control in Smart Grids. IEEE Journal on Selected Areas in Communications, V. 31, No. 7, pp. 1268-1280.

Safdarian, A., Fotuhi-Firuzabad, M., Lehtonen, M. (2014) Integration of Price-Based Demand Response in DisCos' Short-Term Decision Model. IEEE Transactions on Smart Grid, V. 5, No. 5, pp. 2235-2245.
Saffre F., Gedge, R. (2010) Demand-side management for the smart grid. In: 2010 IEEE Network Operations and Management Symposium Workshop, pp. 300-303.

Samadi, P., Mohsenian-Rad, H., Schober, R., Wong, V. W. S. (2012) Advanced Demand Side Management for the Future Smart Grid Using Mechanism Design. IEEE Transactions on Smart Grid, V. 3, no. 3, pp. 1170-1180.

Santos, A. Q. O., Almeida, A. B., Lotero, R. C. (2018). Modelo para gerenciamento energético de microrrede em modo conectado. Congresso Brasileiro de Automática 2018, pp. $1-8$.

Varian, H. R. (2005). Intermediate Microeconomics: A modern Approach. W. W. Norton \& Company.

Zambroni de Souza et. al. (2017). Overview on Microgrids: Technologies, Control, and Communications. In: Azzopardi, B. Sustainable Development in Energy Systems, Springer. 